liver and spleen stiffness in 3 patient groups. Group 1: HIV and $\mathrm{NCPH}$, defined as the presence of portal hypertension manifestations in the absence of cirrhosis; Group 2: HIV and past ddI exposure (without known NCPH), Group 3: HIV and no history of liver disease. Groups were matched for age, HIV chronicity and antiretroviral treatment (including cumulative ddI exposure in Groups 1 and 2). Clinical and demographic information was collected. Differences in liver and spleen stiffness (in $\mathrm{kPa}$ ) between groups were analysed using the Mann-Whiney U test

Results 25 patients were recruited (Group 1: $n=11$, Group 2: $n=5$, Group 3: $n=9$ ). Patients were well matched for age, HIV chronicity and all had HIV RNA levels $<20$ copies $/ \mathrm{mL}$. Cumulative ddI exposure in Groups 1 and 2 was 56 and 53 months respectively $(p=0.91)$. Median (IQR) ARFI liver and spleen stiffness in Group 1, 2 and 3 was 5.5 (4.8-9.8), 4.3 $(4.0-5.3)$ and $4.8(3.8-5.2) \mathrm{kPa}(\mathrm{p}=0.031)$ and $46.3(29.5-$ 143.2), $21.3(14.6-26.8)$ and $18.3(14.6-21.6) \mathrm{kPa}(\mathrm{p}=0.001)$ respectively. Liver and spleen stiffness were both significantly higher in NCPH vs ddI-exposed $(p=0.019$ and $p=0.005)$ and ddI-unexposed controls $(p=0.038$ and $p<0.001)$. Spleen stiffness was more effective than liver stiffness at predicting $\mathrm{NCPH}$, AUROC 0.812 vs 0.948 . Combining the two variables improved the diagnostic performance, AUROC 0.961. The optimal cut-off for predicting NCPH using splenic stiffness was $25.4 \mathrm{kPa}$, with sensitivity 91\%, specificity 93\%, PPV 91\%, NPV 93\%, positive likelihood ratio 12.73 , negative likelihood ratio 0.10. Spleen and liver stiffness scores were strongly correlated ( $\mathrm{p}=0.000495 \%$ CI 18,59$)$.

Conclusions Elevated spleen stiffness is observed in HIV patients with NCPH and can be quantified easily using ARFI with high diagnostic accuracy. Novel strategies such as ARFI for longitudinal monitoring of patients with HIV and NCPH should be considered.

\section{PWE-074 HOSPITALITY DISCHARGE FOR ALCOHOL RELATED PROBLEMS IN NORTH OF ITALY: A SIXTEEN - YEARS PERIOD}

Erik Rosa-Rizzotto*, Diego Caroli, Mario Saia, Laura Scribano, Laura Peraro, Serena Vicario, Salvatore Lobello, Franca De Lazzari. St. Anthony Hospital, Padova, Italy

\subsection{6/gutjpl-2018-BSGAbstracts.216}

Background and aims WHO (2014) estimates a remarkable decline in per capita pure alcohol consumption in Italy, dropped from 18.1 to $7.1 \mathrm{lt}$ in the period 1970-2013. Despite this, Italian Report on Alcohol 2016 showed an increase in drinking outside meals and a rise in consumption and binge drinking among young people (18-24, 14-17), particularly in males. The impact of these drinking styles on hospitalisation is still under-researched. This study aims to evaluate the trends of hospitality discharge for alcohol-related liver disease in the period 2000-2016 in Veneto Region in North Eastern Italy (4.8 million inhabitants).

Method Retrospective cohort analysis based on Veneto Region anonymous computerised database of hospital discharges between 2000 and 2016. All Veneto residents discharge records with principal diagnosis of alcohol-related liver disease (cod. ICD9-CM: 571.0, 571.1, 571.2, 571.3) were included in the study. The principal diagnosis was chosen as it is considered the primary reason for hospital admission. Standardised Hospitalisation Ratio (SHR) per five-year age group (ref. pop. Veneto 2008) was calculated and expressed per 100.000 population.

Results Over the period 2000-2016, 28.968 hospital admissions for alcohol-related diseases were recorded. Most part of subjects were males (74\%) with a SHR more than double compared to females (53.3 vs. 18; OR:2.96; CI 95\%:2.89$3.04 \mathrm{p}<0,05)$. The longitudinal analysis of the hospitalisation trend shows a $7 \%$ increase on average age in both sexes (from $58.8 \pm 9,2$ to $62.6 \pm 9,6$ ) and a substantial decrease of $66 \%$ in SHR (X2 trend: 3933,326). In the last year of observation SHR tends to 19.5 , and the greater risk for males is confirmed (30.2 vs. 8.8; OR:3.51; CI 95\%:3.05-4.10; $\mathrm{p}<0.05)$. Considering the age groups, the highest decline in SHR can be found in the ranges 45-64 (from 69.2 to 34.1) and $>65$ (from 69.3 to 26.8). Interestingly, SHR shows a slightly rising trend in the group 25-44 between 2013 and $2016(\mathrm{p}<0.05)$.

Conclusion In Veneto Region, the reduction in alcohol intake over the last 30 years has lead to a marked decrease in hospitalisation for alcohol-related diseases. However, the changes in drinking styles occurred in the age range 25-44 may explain the upward SHR trend between 2013 and 2016. Thus, in the next few years it is likely to expect an increase in hospitalisation in this age group. Public Health strategies are needed to address the new styles of alcohol consumption, especially in young people.

\section{FEMALE}
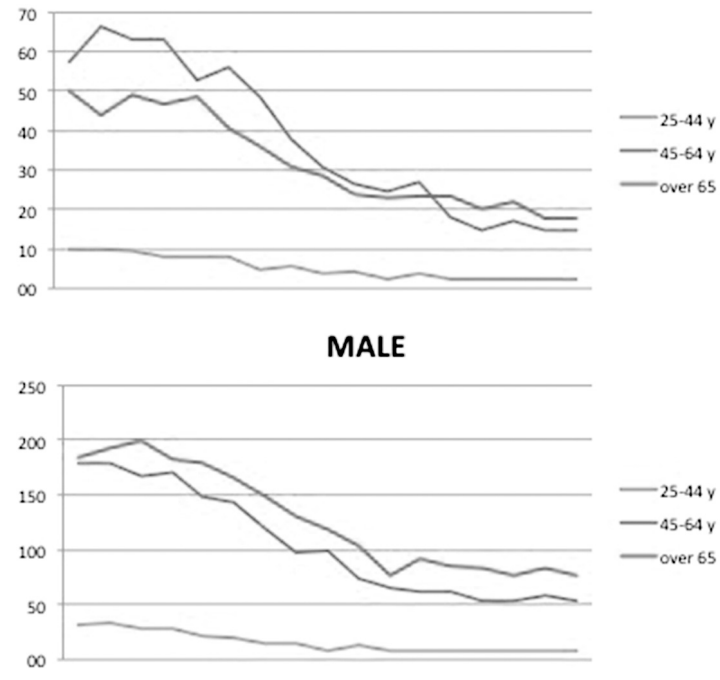

Abstract PWE-074 Figure 1

\section{PWE-075 MANAGING NAFLD VIA A MULTIDISCIPLINARY CLINIC APPROACH IMPROVES LIVER HEALTH AND IS COST EFFECTIVE}

${ }^{1}$ Jeremy Cobbold, ${ }^{2}$ Kenzo Motohashi, ${ }^{2}$ Tom Marjot, ${ }^{1}$ Amelia Shard, ${ }^{1}$ Mark Ainsworth, ${ }^{2}$ Jeremy Tomlinson, ${ }^{2}$ Ahmad Moolla. ${ }^{1}$ Department of Hepatology, Oxford University Hospitals NHS Foundation Trust, UK; ${ }^{2}$ Oxford Centre for Diabetes, Endocrinology and Metabolism, and NIHR Oxford BRC, University of Oxford, UK

10.1136/gutjnl-2018-BSGAbstracts.217

Introduction Non-Alcoholic Fatty Liver Disease (NAFLD) is the hepatic manifestation of metabolic syndrome and is tightly associated with type 2 diabetes (T2DM). Management centres around weight loss and therapies for diabetes and 